\title{
Group problem-solving skills training for self-harm: randomised controlled trial
}

Carmel McAuliffe, Breda C. McLeavey, Tony Fitzgerald, Paul Corcoran, Bernie Carroll, Louise Ryan, Brian O'Keeffe, Eva Fitzgerald, Portia Hickey, Mary O'Regan, Jillian Mulqueen and Ella Arensman

\section{Background}

Rates of self-harm are high and have recently increased. This trend and the repetitive nature of self-harm pose a significant challenge to mental health services.

\begin{abstract}
Aims
To determine the efficacy of a structured group problemsolving skills training (PST) programme as an intervention approach for self-harm in addition to treatment as usual (TAU) as offered by mental health services.
\end{abstract}

\section{Method}

A total of 433 participants (aged 18-64 years) were randomly assigned to TAU plus PST or TAU alone. Assessments were carried out at baseline and at 6-week and 6-month follow-up and repeated hospital-treated self-harm was ascertained at 12-month follow-up.

\section{Results}

The treatment groups did not differ in rates of repeated selfharm at 6-week, 6-month and 12-month follow-up. Both treatment groups showed significant improvements in psychological and social functioning at follow-up. Only one measure (needing and receiving practical help from those closest to them) showed a positive treatment effect at 6week $(P=0.004)$ and 6-month $(P=0.01)$ follow-up. Repetition was not associated with waiting time in the PST group.

\section{Conclusions}

This brief intervention for self-harm is no more effective than treatment as usual. Further work is required to establish whether a modified, more intensive programme delivered sooner after the index episode would be effective.

\section{Declaration of interest}

None.
Rates of self-harm have shown a steady increase in recent years, particularly in men. ${ }^{1}$ Self-harm is the most important risk factor for suicide ${ }^{2-4}$ and the risk of suicide is further increased among those who self-harm repeatedly, particularly among females. ${ }^{5}$ Rates of repeated self-harm are significant and increasing. ${ }^{6}$ Yet there has been a lack of research evidence of effective treatment interventions for self-harm, ${ }^{7,8}$ which limits the power of treatment guidelines. ${ }^{9,10}$ Poorer problem-solving ability has been found among people engaging in self-harm, ${ }^{11-15}$ particularly those who self-harm repeatedly. ${ }^{16-18}$ There is substantial evidence that problem-solving ability mediates the relationship between stress and self-harm, whereby individuals with poor problem-solving ability under chronic stress are more likely to become hopeless and/or suicidal. ${ }^{19-22}$ Evidence also suggests that good problemsolving protects against self-harm, independently of depression or hopelessness levels. ${ }^{16}$ Among people who self-harm, coping responses characterised by greater passivity and avoidance are associated with an increased risk of repeated self-harm. ${ }^{18}$

Promising results have been found for problem-solving therapy in reducing repetition of self-harm. ${ }^{7-8,23}$ In an early study of interpersonal problem-solving skills training, 39 patients who had self-poisoned were randomly assigned to five sessions of individual interpersonal problem-solving skills training or to a brief problem-oriented approach. Although similar improvements were found in levels of hopelessness and presenting problems for both treatment conditions, those assigned to interpersonal problem-solving skills training had a lower rate of repetition at 12-month follow-up compared with the control group. ${ }^{24}$ The difference in repetition between treatment groups was not statistically significant however as, like many of the early trials, the sample size was too small. A later trial involving 120 adults who had recently self-harmed reported a significantly lower repetition rate among those assigned to a cognitive therapy arm comprising ten out-patient cognitive therapy sessions that included a problem-solving component, compared with usual care. ${ }^{25}$ The investigators also reported a significant improvement in self-reported levels of depression and hopelessness, but there was no difference between treatment conditions on rates of suicidal ideation. Generalisation of the study outcomes is difficult because of the high self-harm repetition rate in the control group. Another trial $^{26}$ examined a 12-session cognitive-behavioural therapy (CBT) programme with 90 adolescents and young adults (aged 15-35 years) who had recently engaged in self-harm. The programme was based on a model of maintenance factors of self-harm drawing on the assumption that they can be modified by adjusting negative thinking and problem-solving deficits. The authors reported a significant reduction in repetition of self-harm in the CBT group. Even though the study outcomes support the efficacy of brief CBT for self-harm, it is not clear whether the outcomes can be generalised because of the relatively young target population and the pattern of frequent self-harm repetition prior to enrolment in the study. The failure of an earlier trial using a manual-assisted cognitive therapeutic approach to demonstrate a reduction in repeat episodes, ${ }^{27}$ in which over a third of the active treatment sample received a treatment manual alone without any treatment sessions, suggests that reliance purely on a self-help approach among repeaters of self-harm is ineffective in reducing repetition..$^{28}$ Again, generalisation of the study outcomes was hampered by only including patients with a history of self-harm acts.

In the present study, the effectiveness of a brief group problem-solving skills training (PST) programme for self-harm was examined among both younger and older adults who have self-harmed, and including those with and without a history of self-harm. When the PST programme was previously compared against standard care in the treatment of individuals who have self-poisoned using individual psychotherapy, lower rates of repeated self-harm were reported in the PST group. ${ }^{24}$ The intervention was based on a problem-solving model of self-harm and its repetition, originally developed for the treatment of 
depression. ${ }^{29}$ Compared with treatment as usual (TAU) alone, the PST programme was expected to be significantly more effective in: (a) reducing the rate of repetition of self-harm and suicidal ideation; (b) improving psychological and social functioning as assessed by standardised measures of interpersonal problemsolving skills, self-efficacy and perceived social support; and (c) reducing levels of depression, anxiety, hopelessness and impulsivity.

\section{Method}

\section{Design}

Following initial assessment, participants were randomly assigned to treatment conditions on the basis of a computer-generated sequence of numbers. Allocation was concealed using sealed opaque envelopes. Randomisation was stratified according to the gender and repeater status of participants as well as the study site at which participants were recruited. Participants were randomly assigned to either six sessions of group PST in addition to TAU (standard care) as offered by mental health services or to TAU only. The trial was approved by the clinical research ethics committee of the Cork University Teaching Hospitals and the HSE Mid-Western Area Regional Ethics Committee.

\section{Participants}

Consecutive patients aged 18-64 were included in the trial if, during the previous 3 days, they had engaged in self-harm defined according to the definition devised by the World Health Organization (WHO) Working Group of the WHO/EURO Multicentre Study on Suicidal Behavior as 'an act with non-fatal outcome, in which an individual deliberately initiates a nonhabitual behaviour that, without intervention from others, will cause self-harm, or deliberately ingests a substance in excess of the prescribed or generally recognized therapeutic dosage, and which is aimed at realizing changes which the subject desired via the actual or expected physical consequences. ${ }^{30}$ All participants received a psychiatric review by a liaison psychiatrist in line with standard practice in all recruiting emergency departments and acute psychiatric units. On the basis of the psychiatric review notes, patients were excluded from the trial if they had a history of psychosis, intellectual disability, sensory disability or organic cognitive impairment; were currently alcohol or drug dependent; were in prison at the time of the episode; or were not living at a fixed abode. The baseline assessment schedule included the Short Alcohol Dependent Data questionnaire (SADD) ${ }^{31}$ a 15 -item measure of present state dependence among adults. Only those with a diagnosis of alcohol or drug dependence or who scored above the cut-off for dependence on the SADD were excluded.

Recruitment was conducted at two trial sites (Cork and Limerick) between November 2001 and March 2005. The Cork site comprised the emergency departments of Cork University Hospital, Mercy University Hospital and South Infirmary-Victoria University Hospital and the acute psychiatric units at Cork University Hospital and Mercy University Hospital. The Limerick site comprised the emergency department and the acute psychiatric unit at the Mid-Western Regional Hospital. The trial was stopped when the target number was reached. After trial commencement the eligibility criteria were broadened to include patients self-harming on acute psychiatric units (with or without presentation to the emergency department) at the recruiting hospitals in order to increase recruitment.

\section{Procedure}

At each of the recruitment centres, informed written consent was obtained by trained research officers from eligible patients who had engaged in self-harm within the previous 3 days prior to initial assessment and randomisation. Participants were then assessed either at the recruitment site or at home using a structured assessment schedule. The first section, which assessed characteristics of the index episode and symptoms, was administered within 3 days of the index episode. Where possible, the remainder of the schedule was administered at the same time but where circumstances did not allow for this, arrangements were made to complete the assessment within 2 weeks of the index episode. Psychological, behavioural and social characteristics of participants were assessed at baseline, 6-week (i.e. post-treatment) and 6-month follow-up, using the instruments outlined in Appendix 1.

As part of the consent sought, all participants were encouraged to identify a significant other (for example a friend or family member) who would support their initial connection with the programme. Following randomisation, significant others were informed by letter that they had been nominated to support involvement in the treatment programme (PST or TAU groups) and were encouraged to do so in practical ways such as transport to treatment sessions or through moral support and encouragement. Following recruitment all participants (PST and TAU groups) were contacted by telephone on a weekly basis to minimise pretreatment attrition.

\section{Six-week follow-up}

At the end of treatment (following completion of each PST group programme), participants in both groups completed a post-assessment schedule, which was broadly similar to the baseline assessment (Appendix 1).

\section{Six-month follow-up}

Six months after the treatment ended, participants in both groups completed a shortened version of the post-assessment schedule (Appendix 1).

\section{Twelve-month follow-up}

For all 433 participants, researchers, masked to participant treatment allocation, manually checked the emergency department records of local hospitals in order to identify presentations as a result of repeated self-harm in the 12 -month period after participants' index acts.

\section{Outcome measures}

The primary outcome measure was the proportion of participants in each group who repeated self-harm during the follow-up period. At 6-week and 6-month follow-up, this was assessed by self-report of any repeated self-harm (whether hospital treated or not), whereas at 12-month follow-up the outcome was based on repeated self-harm leading to hospital presentation. Although there was no centralised mechanism for identifying cases of suicide, those detected via hospital records were included in the 12-month follow-up. Secondary outcome measures included suicidal ideation, depression, hopelessness, anxiety, impulsivity, self-efficacy, problem-solving (process and outcome measures) and social life (Appendix 1).

\section{Intervention}

\section{Problem-solving skills training}

The PST programme (B. McLeavey, personal communication, 2001) consisted of six $2 \mathrm{~h}$ closed group sessions, held weekly, of structured, manualised interpersonal problem-solving skills training, facilitated 
by a trained therapist and a co-therapist. A 'Practice at Home Journal' was provided for participants to carry out homework assignments using their own interpersonal problems (B. McLeavey, personal communication, 2001). Treatment fidelity was ensured by strict adherence to the skills training manual, completion of a session-adherence self-report worksheet by therapists at the end of each weekly session and weekly supervision by a research psychologist who had delivered the training to the therapists. Supervision included screening of session-adherence self-report worksheets. The programme was held in a central communitybased venue in both trial sites (Cork and Limerick). To minimise attrition rates, problem-solving therapists made routine betweensession telephone calls to participants in the PST group to remind them of the date and time of their next appointment.

\section{Treatment as usual}

Treatment as usual involved assessment by mental health professional staff and by crisis nurses. Psychosocial assessment of all patients was carried out by a psychiatrist (liaison psychiatry or mental health team) to determine mental health needs and level of risk to self or others. Patients who had no contact with mental health services during the previous year and not requiring referral on to mental health acute or community-based services were referred to the crisis nurse service for further psychosocial assessment and suicide risk assessment. A collaborative management plan of care, including a problem-solving approach and relapse prevention techniques, was agreed between the crisis nurse and the patient. Those who were referred on by the psychiatrist to mental health acute or community-based services were commonly offered pharmacological treatment and review by the mental health team and less frequently counselling or psychotherapy.

\section{Power analysis}

Based on the power calculation, 219 participants were required in each treatment condition of the trial in order for the study to have $80 \%$ power to identify a reduction from $20 \%$ to $10 \%$ in the proportion who repeated self-harm as being statistically significant at the 5\% significance level. The expected effect was based on an earlier smaller trial of problem-solving therapy in the same setting in which the rate of repeated self-harm among those assigned to PST was $11 \%$ at 12 months v. $25 \%$ among those assigned to standard care. ${ }^{24}$

\section{Statistical analysis}

For the primary outcome measure (repeated self-harm), separate binary regression models were estimated for each follow-up period. These models were estimated for participants who provided selfreport data on the outcome measure at 6-week (313 of 354) and 6-month (234 of 326) follow-up. The model for the outcome of hospital-treated repeated self-harm was based on all 433 participants. The covariates included were the treatment condition and whether the participant had a history of self-harm prior to the index act. For the PST group the association between the waiting time (time from the index episode to starting PST) and repeated self-harm was examined using a binary regression that included the logtransformed waiting time and history of self-harm prior to the index episode.

For each of the other outcome measures, treatment effect (PST $v$. TAU) was estimated using a linear mixed-effects model, ${ }^{32}$ which utilised all available data from baseline and follow-up. Treatment effect was not assessed for the problem-solving skills measures, Means-Ends Problem-Solving Procedure (MEPS) ${ }^{33}$ and the
Optional Thinking test (OT), ${ }^{34}$ as these measures were only assessed at 6-week follow-up and were incomplete for $45 \%$ of participants. The linear mixed-effects models included a random intercept to allow for correlations between repeated measures on the same individual. The covariates included were the treatment condition, follow-up period and the interaction of treatment condition and follow-up period. The interaction term allows the effect of treatment to differ between 6-week and 6-month follow-up. Data were analysed using Stata version 12.1 for Windows.

\section{Results}

During the recruitment phase, 2661 patients presenting with selfharm were screened for the trial (Fig. 1). Over half of the patients screened $(1527,57 \%)$ were ineligible. Reasons for ineligibility included being alcohol dependent $(16 \%)$, outside the age range $(13 \%)$, with a current or previous psychosis (8\%), living outside the trial area $(7 \%)$, drug dependent $(3 \%)$, not medically fit within the required time to complete the initial assessment $(2 \%)$, of no fixed abode (2\%) and having an intellectual disability (1\%). Of the 1134 eligible patients (43\%), $433(38 \%)$ were randomised whereas $701(62 \%)$ refused to participate. Most of the 433 participants $(313,72 \%)$ were recruited from the local hospital emergency departments and the remaining 120 (28\%) were recruited from acute mental health in-patient units. A total of 222 patients were randomised to group problem-solving skills training (PST group) and 211 were randomised to treatment as usual (TAU group). The median length of time from index episode to starting PST was 40 days.

Baseline characteristics of participants in each treatment group are reported in Table 1 . Participants assigned to TAU $(n=211)$ were similar to those in the PST group $(n=222)$ with regard to all measures. A higher proportion of those assigned to TAU had self-harmed using an overdose at index episode.

\section{Outcome measures at baseline, post-assessment and follow-up}

The treatment groups did not differ in rates of repeated self-harm at 6-week, 6-month and 12-month follow-up (Table 2). Similar numbers of repeat presentations to hospital were recorded for participants in both treatment conditions in the 12 months following the index presentation. For the 54 of the 222 participants assigned to PST who made a repeat self-harm presentation to hospital, $38(70.4 \%)$ represented once, $9(16.7 \%)$ represented twice, $2(3.7 \%)$ represented 3 times, $3(5.6 \%)$ represented 4 times, $1(1.9 \%)$ represented 8 times and $1(1.9 \%)$ represented 13 times. For the 50 of the 211 participants assigned to TAU who represented because of self-harm, $30(60.0 \%)$ represented once, $10(20.0 \%)$ represented twice, $3(6.0 \%)$ represented 3 times, $3(6.0 \%)$ represented 4 times, $3(6.0 \%)$ represented 5 times and $1(2.0 \%)$ represented 18 times. Three participants were known, based on hospital records, to have died by suicide during the 12-month follow-up period (two in the TAU group and one in the PST group). Repetition was not associated with waiting time in the PST group.

Compared with those in the TAU group, participants in the PST group did not show a significantly different change on any of the secondary outcome measures at 6-week or 6-month follow-up, with the exception of the Practical Support subscale from the Social Life Scale ${ }^{35,36}$ on which there was a positive treatment effect at 6 weeks $(P=0.004)$ and at 6 months $(P=0.01)$. The Practical Support subscale is a measure of the extent to which a person needs practical help and receives practical help from the person closest to them. 


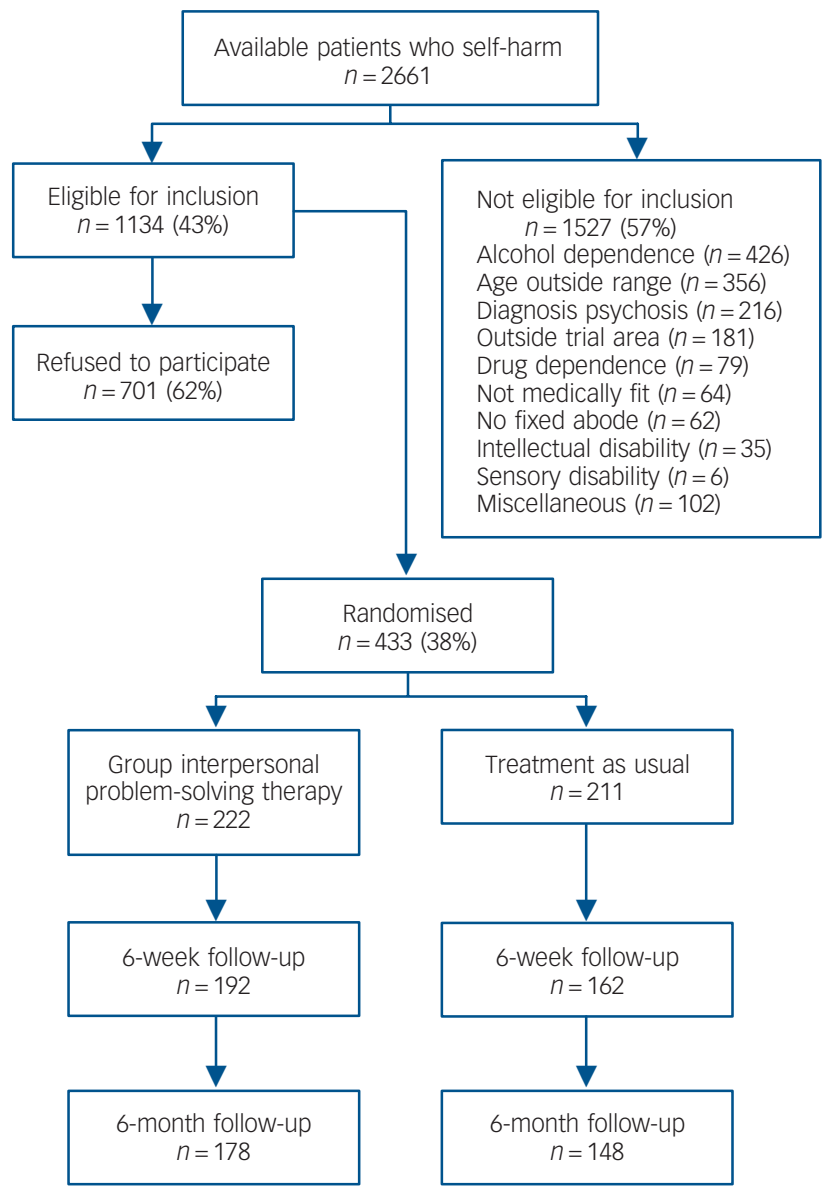

Fig. 1 Flow of participants through the trial.

\section{Drop-out rates}

There was a significant difference in the rate of drop-out from the trial (in terms of those failing to present at follow-up) between the two groups. Drop-out rates were higher in the TAU group 23\% $v$. $14 \%$ in the PST group $(P=0.009)$ at 6 -week assessment and 30\% in the TAU group $v .20 \%$ in the PST group $(P=0.02)$ at 6 -month follow-up. There was no difference in baseline measurement between those who attended the 6-week assessment and those who did not, with the exception of scores on the Beck Anxiety Inventory $^{40}$ (mean 23.5 v. 26.8 respectively, $P=0.04$ ). There was no difference in baseline measurements between those who attended the 6-month follow-up and those who did not.

\section{Adherence with group interpersonal problem-solving skills training}

A relatively high rate of adherence to treatment was found among those assigned to PST. Almost half of those assigned to PST (103, $46.4 \%$ ) attended all 6 therapy sessions. Over two-thirds of those assigned to PST $(153,68.9 \%)$ attended three or more treatment sessions. Most of the attrition occurred prior to the patients' first session, with 43 patients (19.4\%) failing to attend any sessions. When adherence was examined among those who attended at least one treatment session, 103 (57.5\%) of patients attended all six sessions and $153(85.5 \%)$ attended three or more PST sessions. Unfortunately, as a result of the wide range of treatments received by those assigned to TAU and the wide range of settings in which these treatments were delivered it was not possible to ascertain adherence to treatment for this group.

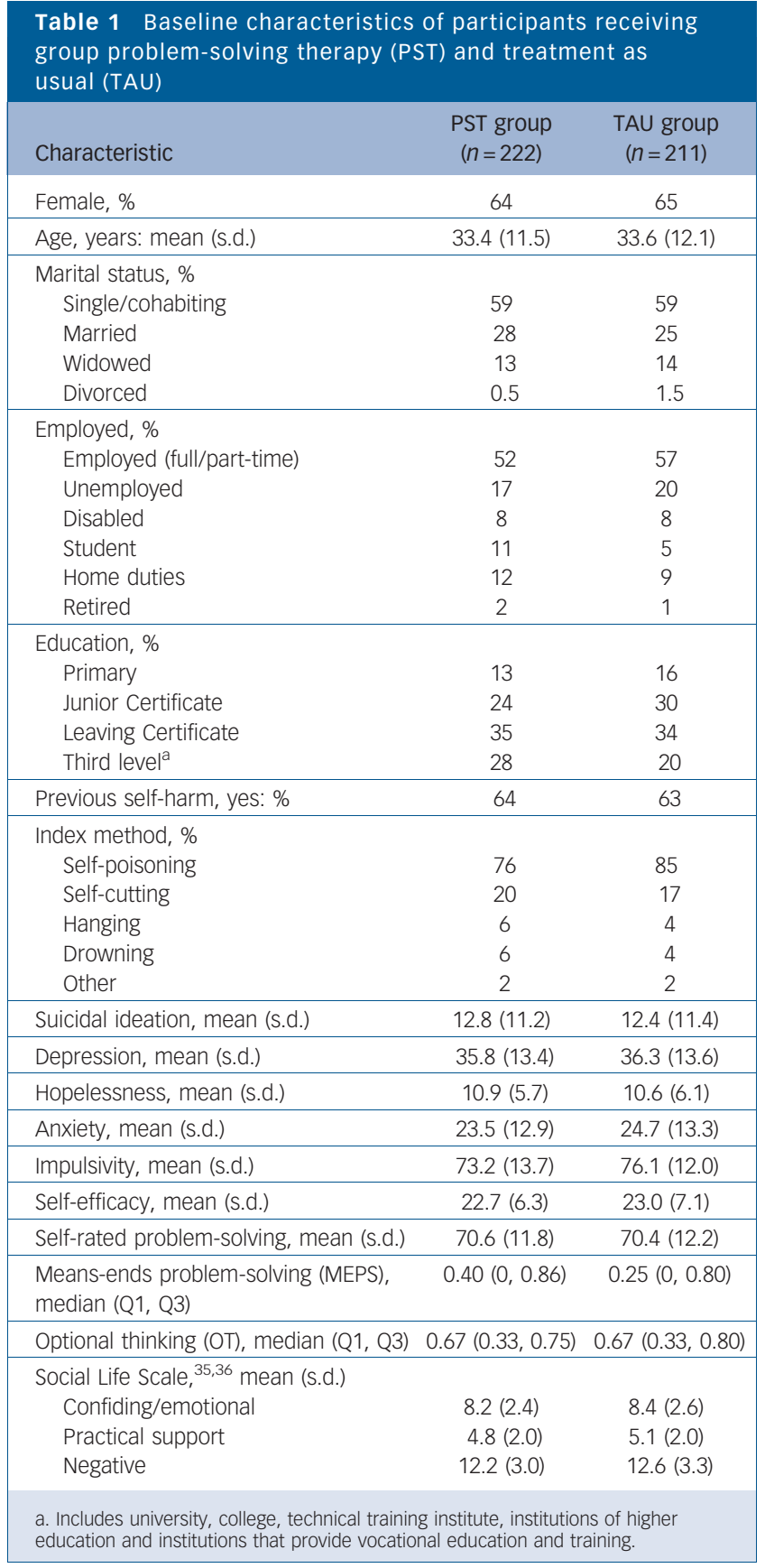

\section{Discussion}

\section{Main findings}

The brief group problem-solving skills training programme described was designed to enhance standard care following an episode of medically treated self-harm. The main trial hypothesis, that PST in addition to standard care would be significantly more effective in reducing repetition of self-harm than TAU alone, was not supported. Compared with TAU alone, those who received PST in addition to standard care did not show significantly greater improvement in psychological and social functioning or significantly greater reductions in depression, anxiety, hopelessness or impulsivity. In fact, no significant differences were found between participants in the PST and TAU condition on any of the outcome measures examined except the Practical Support subscale of the Social Life Scale ${ }^{35,36}$ on which participants in the PST condition showed a significantly greater improvement at 6 weeks and at 6 months. 


\begin{tabular}{|c|c|c|c|c|}
\hline & \multicolumn{2}{|c|}{ Group } & \multirow{2}{*}{\multicolumn{2}{|c|}{ Treatment effect, PST $v$. TAU }} \\
\hline & \multirow{2}{*}{$\begin{array}{l}\text { Problem-solving skills } \\
\text { training (PST) }(n=222)\end{array}$} & \multirow{2}{*}{$\begin{array}{l}\text { Treatment as usual (TAU) } \\
\qquad(n=211)\end{array}$} & & \\
\hline & & & Mean $(95 \% \mathrm{Cl})^{\mathrm{b}}$ & $P$ \\
\hline \multicolumn{5}{|c|}{ Repeated self-harm during follow-up, \% } \\
\hline At 6 weeks $(n=313)$ & 13.5 & 19.0 & 0.71 (0.43 to 1.18$)$ & 0.19 \\
\hline At 6 months $(n=234)$ & 30.5 & 24.5 & 1.23 (0.81 to 1.87$)$ & 0.33 \\
\hline At 12 months $(n=433)^{c}$ & 24.3 & 23.7 & $1.02(0.73$ to 1.42$)$ & 0.92 \\
\hline \multicolumn{5}{|c|}{$\begin{array}{l}\text { Scales, mean }(95 \% \mathrm{Cl}), P \\
\text { Beck Scale for Suicidal Ideation }{ }^{37}(n=424)\end{array}$} \\
\hline Change at 6 weeks & $-8.4(-10.1$ to -6.7$),<0.001$ & $-6.3(-8.1$ to -4.5$),<0.001$ & $-2.1(-4.5$ to 0.4$)$ & 0.09 \\
\hline Change at 6 months & $-8.3(-9.9$ to -6.6$),<0.001$ & $-7.4(-9.3$ to -5.6$),<0.001$ & $-0.8(-3.3$ to 1.6$)$ & 0.51 \\
\hline \multicolumn{5}{|c|}{ Beck Depression Inventory ${ }^{38}(n=433)$} \\
\hline Change at 6 weeks & $-17.5(-20.2$ to -15.4$),<0.001$ & $-16.3(-18.8$ to -13.7$),<0.001$ & $-1.5(-5.0$ to 2.0$)$ & 0.40 \\
\hline Change at 6 months & $-18.3(-20.8$ to -15.8$),<0.001$ & $-17.5(-20.1$ to -14.9$),<0.001$ & $-0.8(-4.4$ to 2.8$)$ & 0.67 \\
\hline \multicolumn{5}{|c|}{ Beck Hopelessness Scale ${ }^{39}(n=433)$} \\
\hline Change at 6 weeks & $-4.1(-5.1$ to -3.1$),<0.001$ & $-3.3(-4.4$ to -2.3$),<0.001$ & $-0.7(-2.2$ to 0.7$)$ & 0.32 \\
\hline Change at 6 months & $-3.8(-4.8$ to -2.8$),<0.001$ & $-3.4(-4.5$ to -2.3$),<0.001$ & $-0.4(-1.9$ to 1.1$)$ & 0.58 \\
\hline \multicolumn{5}{|c|}{ Beck Anxiety Inventory ${ }^{40}(n=432)$} \\
\hline Change at 6 weeks & $-9.4(-11.3$ to -7.4$),<0.001$ & $-8.1(-10.1$ to -6.0$),<0.001$ & $-1.3(-4.1$ to 1.5$)$ & 0.37 \\
\hline Change at 6 months & $-9.7(-11.6$ to -7.8$),<0.001$ & $-8.7(-10.7$ to -6.5$),<0.001$ & $-1.0(-3.9$ to 1.8$)$ & 0.48 \\
\hline \multicolumn{5}{|c|}{ Barratt Impulsivity Scale ${ }^{41}(n=414)$} \\
\hline Change at 6 weeks & $-1.0(-2.7$ to 0.8$), 0.27$ & $-2.6(-4.4$ to -0.8$), 0.01$ & $1.7(-0.9$ to 4.2$)$ & 0.20 \\
\hline Change at 6 months & $-1.7(-3.5$ to 0.1$), 0.06$ & $-3.4(-5.3$ to -1.4$),<0.001$ & $1.6(-1.0$ to 4.2$)$ & 0.23 \\
\hline \multicolumn{5}{|c|}{ Generalised Self-efficacy Scale ${ }^{42}(n=431)$} \\
\hline Change at 6 weeks & $3.2(2.2$ to 4.2$),<0.001$ & $2.2(1.1$ to 3.2$),<0.001$ & $1.1(-0.4$ to 2.6$)$ & 0.16 \\
\hline Change at 6 months & 3.7 (2.7 to 4.8$),<0.001$ & 2.7 (1.6 to 3.8$),<0.001$ & $1.0(-0.5$ to 2.5$)$ & 0.20 \\
\hline \multicolumn{5}{|c|}{ Self-Rating Problem-Solving Scale ${ }^{43}(n=432)$} \\
\hline Change at 6 weeks & 8.1 (6.1 to 10.1$),<0.001$ & 6.0 (3.8 to 8.3 ), $<0.001$ & $2.1(-0.9$ to 5.1$)$ & 0.17 \\
\hline Change at 6 months & $8.6(6.6$ to 10.7$),<0.001$ & 7.7 (5.4 to 10.0$),<0.001$ & $0.9(-2.1$ to 4.0$)$ & 0.55 \\
\hline \multicolumn{5}{|c|}{ Social Life Scale: confiding/emotional ${ }^{35,36}(n=404)$} \\
\hline Change at 6 weeks & $-0.8(-1.2$ to -0.3$),<0.001$ & -0.5 ( -1.0 to 0.0$), 0.03$ & $-0.3(-0.9$ to 0.4$)$ & 0.45 \\
\hline Change at 6 months & $-0.8(-1.2$ to -0.3$),<0.001$ & $-0.6(-1.1$ to -0.1$), 0.02$ & $-0.2(-0.8$ to 0.5$)$ & 0.66 \\
\hline \multicolumn{5}{|c|}{ Social Life Scale: practical support ${ }^{35,36}(n=403)$} \\
\hline Change at 6 weeks & $0.1(-0.3$ to 0.4$), 0.74$ & $-0.7(-1.1$ to -0.3$),<0.001$ & $0.8(0.3$ to 1.3$)$ & 0.004 \\
\hline Change at 6 months & $-0.1(-0.5$ to 0.3$), 0.55$ & $-0.8(-1.3$ to -0.4$),<0.001$ & $0.7(0.2$ to 1.3$)$ & 0.01 \\
\hline \multicolumn{5}{|c|}{ Social Life Scale: negative ${ }^{35,36}(n=403)$} \\
\hline Change at 6 weeks & $1.0(0.5$ to 1.5$),<0.001$ & $0.2(-0.3$ to 0.8$), 0.40$ & 0.7 (0.0 to 1.5$)$ & 0.06 \\
\hline Change at 6 months & 1.3 (0.8 to 1.8$),<0.001$ & 0.9 (0.3 to 1.5$), 0.004$ & $0.4(-0.4$ to 1.2$)$ & 0.35 \\
\hline
\end{tabular}

Participants in both the PST and TAU groups showed significant improvements on most outcome measures. In both groups, participants improved significantly on 9 out of 11 outcome measures comparing baseline to 6-week follow-up. At 6-month follow-up no further significant changes were observed for these outcome measures, indicating that improvements made in both treatment conditions were maintained over time.

\section{Practical problems with the implementation of design and lack of differential treatment effects}

The theoretical model underlying the experimental treatment (PST) condition was that the development of improved interpersonal problem-solving skills in participants would lead to reduced vulnerability to repeated self-harm. Although significant improvements were found in these skills among participants in both groups, no significant differences were found between those in the PST group and those in the TAU group on any of the outcome measures of problem-solving at 6-week or 6-month follow-up. Several procedural aspects of the trial may have had a therapeutic effect on the patient's condition and therefore may have contributed to the lack of differential treatment outcomes between the groups. As described earlier, participants in both the PST and TAU groups were encouraged to identify a significant other (for example a friend or family member) who would support their initial connection with the treatment programme. Following randomisation, significant others (PST and TAU groups) were informed by letter that their relative or friend had nominated them as their significant other to support their involvement in the treatment programme. Following recruitment, all participants (PST and TAU groups) were contacted by telephone on a weekly basis to minimise pretreatment attrition. The participants themselves were also notified by telephone and by letter of their treatment allocation. Initial and follow-up assessments provided participants in both groups with the opportunity to discuss problems. In cases where participants were discharged from hospital prior to completion of initial assessment and where they could not make their way to the venue for follow-up assessment, home visits were arranged by the researcher. It could be argued that this may have obfuscated differences between the treatment conditions. The possible therapeutic effects of these active intervention aspects should not be underestimated. For example, in an earlier trial by Carter and colleagues ${ }^{44}$ the number of repeat self-harm episodes was significantly reduced in those who received a minimal intervention (eight postcards posted to medically treated individuals who had self-poisoned over a 
12-month follow-up period). Like Carter and colleagues however, the design of the present trial does not allow us to examine the possible mechanism of action of these additional interventions. It can also be argued that the possible therapeutic effects of these procedural aspects occurred in both treatment conditions, which although making them more similar probably did not influence the likelihood of finding a differential treatment effect of the active treatment condition.

\section{Strengths and limitations}

Strengths of this trial include the large sample size and assessment of the primary outcome measure (repeated self-harm) from both participants' self-report and researchers' independent ascertainment. Although significant improvements in outcome measures at follow-up were found among patients in both groups and a number of explanations can be offered for the lack of differences in outcomes between PST and TAU, additional limitations of the trial merit attention.

\section{Differential drop-out rates}

The significantly greater drop out at post-assessment and follow-up of participants assigned to TAU may have masked important differences in treatment outcome at both follow-up periods. Those who failed to attend the 6-week follow-up had significantly higher levels of anxiety at baseline assessment suggesting that they may have been more unwell at follow-up. However, we carried out analyses that included adjustment for baseline values and the findings were highly consistent with those reported in the paper.

\section{Duration, format and waiting time}

A brief group-based interpersonal problem-solving skills training intervention delivered over six sessions may not have been sufficient to significantly reduce repetition among the group of individuals who self-harmed included in the present trial, of whom the majority had a history of self-harm episodes and scored within the severe range on level of depression. The brief interventions tested in trials with patients who have self-harmed (published after our trial commenced) typically include 10-12 treatment sessions. ${ }^{25,26}$ Furthermore, the PST intervention of 6 weeks duration may have been too brief to teach patients skills to interrupt repetition, when considered in the context of the high risk of repetition for the first 12 months following the index episode. This is further supported by negative outcomes of a brief manual-assisted CBT intervention (maximum seven sessions) for patients who had self-harmed, all of whom had a history of self-harm acts. ${ }^{27}$ In contrast, an earlier trial using a brief 12-session CBT intervention lasting approximately 5.5 months, showed positive treatment effects on self-harm repetition and related mental health outcomes favouring the CBT intervention. ${ }^{26}$

Another possible explanation for the lack of differential treatment effects is the group format of the PST programme. A recent review of cognitive-behavioural interventions to reduce suicidal behaviour found that trials where one-to-one CBT was included, and also trials combining individual and group treatment, showed a very significant effect on repetition, whereas studies using group therapy alone did not. ${ }^{23}$

A further important limitation arising from the implementation of a randomised controlled trial investigating group therapy is the potentially significant delay between recruitment and treatment initiation. In the present trial the median length of time between index episode and first PST session was 40 days. Although we found no association with repetition, this almost certainly contributed to the significant attrition prior to PST commencement. Perhaps more importantly this considerable window of time meant that pragmatic measures taken to minimise attrition for all patients (PST and TAU groups) including weekly telephone calls and encouragement from a significant other are likely to have had important treatment effects in both groups and may also explain the lack of differential treatment effects. The clinical profile of participants in the present trial indicates a group that requires more intensive input (incorporating one-to-one and group therapy sessions and long-term treatment approaches), to address patterns of frequent self-harm repetition associated with comorbid psychological and psychiatric problems. ${ }^{25,26}$ Many of the patients who completed the PST intervention indicated the need for more than six treatment sessions, which further supports this explanation. Put more simply the experimental treatment programme in the present trial (PST), may have provided patients with too little too late.

\section{Eligibility}

The most common reason for ineligibility for the trial was alcohol dependence followed by age that was outside the trial age range. Together these comprised half of those excluded from the trial. Given the high risk of repetition among those misusing alcohol ${ }^{45}$ and the high rates of self-harm among girls aged 15-19 years in particular, ${ }^{1}$ this trial failed to evaluate the efficacy of problemsolving skills training in reducing repetition in these subgroups.

\section{Clinical implications}

Our findings indicate that for individuals who self-harm, the majority of whom have a history of self-harm, a brief group problem-solving skills training programme is no more effective than TAU when delivered a median of 40 days following the index episode. In clinical practice this time delay to therapy group initiation would likely be halved as the constraint of randomisation of eligible patients in a parallel trial would be absent. Future trials might reduce potential delays to treatment initiation by broadening the inclusion criteria further, for example time from index episode to initial assessment could be extended up to 1 week. Patients with a history of psychosis but not currently psychotic could also be included. Additional measures to minimise the wait to commence therapy could include offering an initial one-to-one primer session with an overview of the PST programme material or by running the six-session PST programme as a semi-closed rather than a fully closed group on a repeat basis, with each patient repeating the treatment programme over 11 weeks, which could significantly shorten the waiting time. Further work is required to establish whether a modified, more intensive group problem-solving skills training programme delivered sooner following the index episode would be effective.

\footnotetext{
Carmel McAuliffe, BA, HDip, MPhil, MA, PhD, Health Service Executive South/ National Suicide Research Foundation, Cork, and Department of Epidemiology \& Public Health, University College Cork, Ireland; Breda C. McLeavey, BSC, MA, PhD, Health Service Executive South, Department of Psychiatry, Cork University Hospital, Ireland; Tony Fitzgerald, BSC, MSC, MS, SCD, Department of Epidemiology and Public Health, University College Cork, Ireland; Paul Corcoran, BSC, MSC, PhD, National Suicide Research Foundation, Cork, Ireland; Bernie Carroll, BSc, Louise Ryan, BSC, Health Service Executive West, St Joseph's Hospital, Limerick, Ireland; Brian O'Keeffe, BA, DipClinPsychol, Health Service Executive West, Tevere Day Hospital, Limerick, Ireland; Eva Fitzgerald, BA, HDipPsychol, Portia Hickey, BSc, Mary O'Regan, BA, MPhil, National Suicide Research Foundation, Cork, Ireland; Jillian Mulqueen, BA, Health Service Executive West, St Joseph's Hospital, Limerick, Ireland; Ella Arensman, MSc, PhD, National Suicide Research Foundation, and Department of Epidemiology \& Public Health, University College Cork, Ireland

Correspondence: Carmel M. McAuliffe, National Suicide Research Foundation, Western Gateway Building, Western Road, Cork, Ireland. Email: info@nsrf.ie

First received 25 Aug 2011, final revision 3 Sep 2013, accepted 6 Nov 2013
} 


\section{Funding}

This work was supported by funding from the Health Service Executive (HSE) South, HSE Mid-West, the HSE National Office for Suicide Prevention, the Health Research Board and Pobal-Dormant Accounts Fund in Ireland.

\section{Acknowledgements}

The authors wish to thank the therapists, hospital staff, and voluntary research assistant for facilitating this research. They also wish to thank Mr Pat Madden, former Programme Manager Mental Health/Intellectual Disability Services, Health Service Executive South, and Professor Keith Hawton of the Oxford University Centre for Suicide Research for their valuable support

\section{Appendix}

\section{Overview of instruments used to assess} psychological, behavioural and social characteristics assessed at baseline, 6-week post-assessment and 6-month follow-up

Suicide Intent Scale (SIS) ${ }^{46}$

Beck Scale for Suicidal Ideation (BSS)

Beck Depression Inventory (BDI)

Beck Anxiety Inventory (BAl)

Barratt Impulsivity Scale (BIS)

Generalised Self-efficacy Scale (GSS)

Means-Ends Problem-Solving

Procedure (MEPS)

Optional Thinking Test (OT)

Self-Rating Problem-Solving

Scale (SRPS)

Current Problems List ${ }^{43}$

Beck Hopelessness Scale (BHS)

Social Life Scale

$\begin{array}{ccc}\text { Baseline } & 6 \text { weeks } & 6 \text { months } \\ \checkmark^{a} & - & - \\ \checkmark^{a} & \checkmark & \checkmark \\ \checkmark^{a} & \checkmark & \checkmark \\ \checkmark^{a} & \checkmark & \checkmark \\ \checkmark & \checkmark & \checkmark \\ \checkmark & \checkmark & \checkmark \\ \checkmark & \checkmark & - \\ \checkmark & \checkmark & - \\ & & \\ \checkmark & \checkmark & \checkmark \\ \checkmark & \checkmark & \checkmark \\ v^{a} & \checkmark & \checkmark \\ \checkmark & \checkmark & \checkmark\end{array}$

a. Denotes instruments that were administered at baseline within 3 days of the index episode.

\section{References}

1 National Suicide Research Foundation. National Registry of Deliberate Self Harm: Annual Report 2011. National Suicide Research Foundation, 2012.

2 Gunnell D, Frankel S. Prevention of suicide: aspirations and evidence. BMJ 1994; 308: 1227-33.

3 Harris EC, Barraclough B. Suicide as an outcome for mental disorders. A meta-analysis. Br J Psychiatry 1997; 170: 205-28.

4 Sinclair JM, Hawton K, Gray A. Six year follow-up of a clinical sample of selfharm patients. J Affect Disord 2009; 121: 247-52.

5 Zahl DL, Hawton K. Repetition of deliberate self-harm and subsequent suicide risk: Iong-term follow-up study of 11,583 patients. Br J Psychiatry 2004; 185 70-5.

6 O'Loughlin S, Sherwood J. A 20 year review of trends in deliberate self harm in a British town 1981-2000. Soc Psychiatry Psychiatr Epidemiol 2005 40: 446-53.

7 Arensman E, Townsend E, Hawton K, Bremner S, Feldman E, Goldney R, et al. Psychosocial and pharmacological treatment of patients following deliberate self-harm: the methodological issues involved in evaluating effectiveness. Suicide Life Threat Behav 2001; 31: 169-80.

8 Hawton K, Arensman E, Townsend E, Bremner S, Feldman E, Goldney R, et al Deliberate self harm: systematic review of efficacy of psychosocial and pharmacological treatments in preventing repetition. BMJ 1998; 317: 441-7.

9 National Collaborating Centre for Mental Health. Self-harm. The ShortTerm Physical and Psychological Management and Secondary Prevention of Self-Harm in Primary and Secondary Care. National Clinical Practice Guideline Number 16. The British Psychological Society and The Royal College of Psychiatrists, 2004.

10 Royal Australian and New Zealand College of Psychiatrists. Self-harm. Australian Treatment Guide for Consumers and Carers. The Royal Australian and New Zealand College of Psychiatrists, 2009.
11 Linehan MM, Camper P, Chiles JA, Strosahl K, Shearin EL. Interpersonal problem solving and parasuicide. Cog Therapy Res 1987; 11: 1-12.

12 McLeavey BC, Daly RJ, Murray CM, O'Riordan J, Taylor M. Interpersonal problem-solving deficits in self-poisoning patients. Suicide Life Threat Behav 1987; 17: 33-49.

13 Pollock LR, Williams JM. Problem solving and suicidal behavior. Suicide Life Threat Behav 1998; 28: 375-87.

14 Pollock LR, Williams JM. Problem-solving in suicide attempters. Psychol Med 2004; 34: 163-7.

15 Rotheram-Borus MJ, Trautman PD, Dopkins SC, Shrout PE. Cognitive style and pleasant activities among female adolescent suicide attempters. J Consult Clin Psychol 1990; 58: 554-61.

16 Dieserud G, Roysamb E, Braverman MT, Dalgard OS, Ekeberg O. Predicting repetition of suicide attempt. A prospective study of 50 suicide attempters Arch Suicide Res 2003; 7: 1-17.

17 Kehrer CA, Linehan MM. Interpersonal and emotional problem solving skills and parasuicide among women with borderline personality disorder. J Pers Disord 1996; 10: 153-63.

18 McAuliffe C, Corcoran P, Keeley HS, Arensman E, Bille-Brahe U, De Leo D, et al. Problem-solving ability and repetition of deliberate self-harm: a multicentre study. Psychol Med 2006; 36: 45-55.

19 Rudd MD, Joiner T, Rajab MH. Relationships among suicide ideators, attempters, and multiple attempters in a young-adult sample. J Abnorm Psychol 1996; 105: 541-50.

20 Sandin B, Chorot $\mathrm{P}$, Santed MA, Valiente RM, Joiner TE. Negative life events and adolescent suicidal behavior: a critical analysis from the stress process perspective. J Adolesc 1998; 21: 415-26.

21 Schotte DE, Clum GA. Suicide ideation in a college population: a test of a model. J Consult Clin Psychol 1982; 50: 690-6.

22 Schotte DE, Clum GA. Problem-solving skills in suicidal psychiatric patients. J Consult Clin Psychol 1987; 55: 49-54.

23 Tarrier N, Taylor K, Gooding P. Cognitive-behavioral interventions to reduce suicide behavior: a systematic review and meta-analysis. Behav Modif 2008; 32: 77-108.

24 McLeavey BC, Daly RJ, Ludgate JW, Murray CM. Interpersonal problemsolving skills training in the treatment of self-poisoning patients. Suicide Life Threat Behav 1994; 24: 382-94.

25 Brown GK, Ten Have T, Henriques GR, Xie SX, Hollander JE, Beck AT. Cognitive therapy for the prevention of suicide attempts: a randomized controlled trial. JAMA 2005; 294: 563-70.

26 Slee N, Garnefski N, van der Leeden R, Arensman E, Spinhoven P. Cognitive-behavioural intervention for self-harm: randomised controlled trial. Br J Psychiatry 2008; 192: 202-11.

27 Tyrer $\mathrm{P}$, Thompson S, Schmidt U, Jones V, Knapp M, Davidson K, et al. Randomized controlled trial of brief cognitive behaviour therapy versus treatment as usual in recurrent deliberate self-harm: the POPMACT study. Psychol Med 2003; 33: 969-76.

28 Arensman E, McAuliffe C, Corcoran P, Perry IJ. Findings of the POPMACT study. Psychol Med 2004; 34: 1143-4.

29 Nezu AM, Nezu CM, Perri MG. Problem-Solving Therapy for Depression: Theory, Research and Clinical Guidelines. Wiley, 1989.

30 Platt S, Bille-Brahe U, Kerkhof A, Schmidtke A, Bjerke T, Crepet P, et al. Parasuicide in Europe: the WHO/EURO multicentre study on parasuicide. I. Introduction and preliminary analysis for 1989. Acta Psychiatr Scand 1992; 85: $97-104$

31 Raistrick DS, Dunbar G, Davidson RJ. Development of a questionnaire to measure alcohol dependence. Br J Addict 1983; 78: 89-95.

32 Fitzmaurice GM, Laird NM, Ware JH. Applied Longitudinal Analysis. John Wiley \& Sons, 2004

33 Platt JJ, Spivack G, Bloom W. Manual for the Means-Ends Problem-Solving Procedure (MEPS): A Measure of Interpersonal Problem-Solving Skill. Hahneman Medical College and Hospital, Department of Mental Health Sciences, Hahneman Community MH/MR Center, 1975.

34 Platt J, Spivak G. Measures of Interpersonal Cognitive Problem Solving for Adults and Adolescents. Hahnemann Medical College and Hospital, 1977.

35 Stansfeld SA, Marmot MG. Deriving a survey measure of social support: the reliability and validity of the Close Persons Questionnaire. Soc Sci Med 1992; 35: 1027-35.

36 Surtees PG, Wainwright NW, Brayne C. Psychosocial aetiology of chronic disease: a pragmatic approach to the assessment of lifetime affective morbidity in an EPIC component study. I Epidemiol Community Health 2000; 54: $114-22$.

37 Beck AT, Steer RA, Raineri WF. Scale for suicide ideation: psychometric properties of a self-report version. J Clin Psychol 1988; 44: 499-505. 
38 Beck AT, Steer RA, Ball R, Ranieri W. Comparision of Beck Depression Inventories -IA and -II in psychiatric outpatients. J Pers Assess 1996; 67: 588-97

39 Beck AT, Weissman A, Lester D, Trexler L. The measure of pessimism: the hopelessness scale. J Consult Clin Psychol 1974; 42: 861-5.

40 Beck AT, Epstein N, Brown G, Steer RA. An inventory for measuring clinica anxiety: psychometric properties. J Consult Clin Psychol 1988; 56: 893-7.

41 Barratt ES. Factor analysis of some psychometric measures of impulsiveness and anxiety. Psychol Rep 1965; 16: 547-54.

42 Schwarzer R, Jerusalem M. Generalized self-efficacy scale. In Measures in Health Psychology: A User's Portfolio: Causal and Control Beliefs (eds J Weinman, S Wright, M Johnston): 35-7. nferNelson, 1995.
43 McLeavey BC. Self-poisoning: a study of psychological characteristics and an alternative treatment method. Department of Applied Psychology, University College Cork, doctoral dissertation, 1986.

44 Carter GL, Clover K, Whyte IM, Dawson AH, D'Este C. Postcards from the EDGE project: randomised controlled trial of an intervention using postcards to reduce repetition of hospital treated deliberate self poisoning. BMJ 2005; 331: 805

45 Foote B, Smolin Y, Neft DI, Lipschitz D. Dissociative disorders and suicidality in psychiatric outpatients. J Nerv Ment Dis 2008; 196: 29-36.

46 Beck AT, Schuyler D, Herman J. Development of suicidal intent scales. In The Prediction of Suicide (eds AT Beck, HLP Resnik, DJ Lettieri): 45-56. Charles Press, 1974

\section{When a psychiatrist is a patient}

\section{Aashish Tagore}

I am proud to be a psychiatrist. Despite often feeling stigmatised by medical colleagues, I have always derived a great sense of personal reward and satisfaction from my chosen line of work. As psychiatrists, we try to understand how it must feel for our patients, and we strive to treat them with compassion, empathy and humanity. But what happens when you suddenly find yourself on the other side of the fence?

Last year, after a traumatic event, I started experiencing persecutory delusions and was convinced that my life was in grave danger. A descent into full-blown paranoid psychosis ensued, culminating in a hospital admission and treatment with antipsychotic medication.

I was a patient on a ward on which I used to work - my worst nightmare realised. Not only was I embarrassed to be there, the staff with whom I used to work appeared equally embarrassed for me. The pity was written all over their faces. And my own prejudice towards mentally ill patients surfaced. Despite needing acute assessment and treatment, I felt I was for some reason better than or at least different to them. I wanted to distance myself from them - to reassure myself that I was not one of them. But, alas, I was. I was just as unwell, and just as human. I was just as vulnerable and susceptible to mental illness, just as breakable. I was no longer the 'doctor', I was their equal.

After my illness, I felt a sense of deep-seated shame and guilt and came to view myself as 'weak-minded'. This 'self-stigmatisation', where the stigmatised person relates to negative attitudes expressed by others towards themselves and their illness, is debilitating if you share in the negativity surrounding you and view such attitudes as 'understandable', where is the motivation to fight them? Other people's - including my psychiatrist's - wishes to conceal this episode have only served to compound and perpetuate my own sense of shame and embarrassment. Of course, they are concerned about my career. My psychiatrist attempted to minimise the psychotic quality and severity of my illness - does he, too, anticipate discrimination against a fellow doctor with a history of mental illness?

I am relieved to say that currently I am enjoying a period of sustained mental stability, which I can only hope continues ad infinitum. so why did I share my experience of psychotic illness? I surmised that all my anti-stigma support for my patients would not mean a thing unless I was willing to practise as I preach. I would like to think this decision will come to be viewed as one of personal dedication to the cause of de-stigmatising mental illness, and that the whole experience will help me become a better psychiatrist. 